\title{
Gremlin-1 suppression increases BMP-2-induced osteogenesis of human mesenchymal stem cells
}

\author{
KONGZU HU, HEYAN SUN, BINJIE GUI and CONG SUI
}

Department of Orthopaedics, The First Affiliated Hospital of Anhui Medical University, Hefei, Anhui 230032, P.R. China

Received December 16, 2015; Accepted January 23, 2017

DOI: $10.3892 / \mathrm{mmr} .2017 .6253$

\begin{abstract}
Previous research focusing on rodent cells and animal models has demonstrated that gremlin-1 antagonizes bone morphogenetic proteins (BMPs) in order to suppress osteogenesis. However, the impact of gremlin-1 on osteogenesis in human bone marrow-derived mesenchymal stem cells (MSCs) remains unknown. The aim of the present study was to test the effects of gremlin-1 on viability and in vitro BMP-2-induced osteogenic differentiation of human bone marrow-derived mesenchymal stem cells (MSCs). Gremlin-1-specific small interfering RNA (siRNA) inhibited gremlin-1 mRNA and protein expression in human MSCs. The mRNA expression levels of osteoblastic genes were analyzed using reverse transcription-quantitative polymerase chain reaction, and calcification and enzymatic alkaline phosphatase (ALP) activity assessed the BMP-2-induced osteogenic differentiation of human MSCs. The results indicated that gremlin-1 suppression significantly increased human MSC metabolism and DNA content. The expression levels of osteoblastic genes were also significantly increased by gremlin-1 inhibition. In the gremlin-1-inhibited group, enzymatic ALP activity was significantly increased. In addition, due to BMP-2-inducing osteoblasts, gremlin-1 inhibition increased calcium deposits. The present study indicated that gremlin-1 inhibited the cell viability and osteogenic differentiation of human MSCs and that the suppression of gremlin-1 expression suppressed can increase the cell viability and osteogenic differentiation of human MSCs induced by BMP-2.
\end{abstract}

Correspondence to: Dr Kongzu Hu, Department of Orthopaedics, The First Affiliated Hospital of Anhui Medical University, 218 Jixi Road, Hefei, Anhui 230032, P.R. China

E-mail: hukongzu1974@sina.cn

Key words: gremlin-1, human bone mesenchymal stem cells, cell viability, osteogenesis

\section{Introduction}

Mesenchymal stem cells (MSCs) migrate to the injury site following a fracture and differentiate into osteoblasts, which produce the bone matrix and repair the fractured bone (1). Multiple signals, including the potent osteoinductive signal induced by bone morphogenetic proteins (BMPs) regulate this process (2-4). It has been reported that BMP-2, 4, 6, 7 and 9 induce osteogenic differentiation of MSCs in vitro and in vivo (5-11). By binding to type 1 and 2 receptors on the surface of cells, BMPs exert their signals through the activation of intracellular Smad proteins. Then, by interacting with different transcription factors, the activated Smad protein complex regulates the expression of osteoblastic genes (12-14). BMP-mediated osteogenic differentiation is mediated by extracellular BMP antagonists, including noggin, gremlin-1, chordin and follistatin (15-18).

The function of gremlin-1 in BMP-regulated osteogenic differentiation has been previously investigated in rodent cells and animal models and the results have indicated that gremlin-1 is an inhibitor of osteogenesis. Gremlin-1 is a highly conserved glycoprotein with a molecular weight of $27 \mathrm{kDa}$ belonging to the DAN/Cerberus family and was first isolated from Xenopus (19). Gremlin-1 is primarily distributed in the extracellular matrix, but a small amount of gremlin-1 is also distributed in the endoplasmic reticulum (20). Gremlin-1 combines with BMP-2, 4 and 7 and inhibits their association with the BMP receptors on the cell membrane (20). The expression of gremlin-1 was demonstrated to be reduced in tumor cells and contributed to the growth of tumor cells (21). Gremlin-1 is involved in the development of bone and kidney organs, and gremlin-1 mutant mice develop severe limb skeletal deformities (22-25). Mouse bone tissue-specific overexpression of gremlin-1 results in severe symptoms of osteoporosis; however, conditional knockout of gremlin-1 in bone tissue increases bone formation and trabecular bone volume (26). In osteoblasts, overexpression of gremlin-1 reduces the biological activity of BMP-2 and the use of RNA interference to reduce gremlin-1 expression in osteoblasts increases the biological activity of BMP-2 (26). Collectively, these studies demonstrate that the effects of gremlin-1 on BMP-induced osteogenesis of human MSCs remain elusive and further investigation into the involvement of gremlin-1 in osteogenesis of human MSCs is warranted. The present study was conducted to examine the effects of gremlin-1 suppression on cell viability and BMP-2-induced osteogenic differentiation of primary human MSCs in vitro. 


\section{Materials and methods}

Isolation and culture of human MSCs. Bone marrow samples were obtained from 5 donor patients who underwent orthopedic surgery; written informed consent were obtained from all patients. The present study was approved by the Research Ethics Committee of The First Affiliated Hospital of Anhui Medical University (Hefei, China). Mononuclear cells from the bone marrow samples of the 5 patients were separated by centrifugation at $400 \times \mathrm{g}$ for $25 \mathrm{~min}$ at room temperature and Ficoll-Paque medium (GE Healthcare Life Sciences, Chalfont, UK) and then inoculated in MSC growth medium (MGM; high glucose Dulbecco's modified Eagle's medium [DMEM] containing 10\% fetal bovine serum [FBS], $0.29 \mathrm{mg} / \mathrm{ml}$ GlutaMAX, $100 \mathrm{mg} / \mathrm{ml}$ streptomycin, $100 \mathrm{U} / \mathrm{ml}$ penicillin; all from Invitrogen; Thermo Fisher Scientific, Inc., Waltham, MA, USA), and $4 \mathrm{ng} / \mathrm{ml}$ basic fibroblast growth factor (Merck KGaA, Darmstadt, Germany) at a density of $4 \times 10^{5}$ cells $/ \mathrm{cm}^{2}$. Following incubation for 3 days, non-adherent cells were discarded and adherent cells were washed twice using phosphate buffered saline (PBS; Invitrogen; Thermo Fisher Scientific, Inc.) and then cultured in MGM. The cells of each patient were either cultured for further examinations or frozen in liquid nitrogen in $1 \mathrm{ml}$ aliquots 7 days later. Cells were cultured in a $5 \% \mathrm{CO}_{2}$ and incubated at $37^{\circ} \mathrm{C}$ for all experiments. For each patient, all experiments were conducted in either triplicate or quadruplicate on individual cell cultures. The data generated from the individual cell cultures of these patients were collected and presented as combined data in the present study (mean \pm standard deviation of $n=5$ ).

BMP-2 treatment. In order to determine whether BMP-2 induces gremlin-1 mRNA expression in human MSCs, dose-response and time-response studies were conducted. For the dose-response study, human MSCs were inoculated in $35 \mathrm{~mm}$ tissue culture dishes in MGM at a density of $7 \times 10^{3}$ cells $/ \mathrm{cm}^{2}$. The medium was replaced $24 \mathrm{~h}$ later with the basal medium (DMEM containing 10\% FBS, $100 \mathrm{mg} / \mathrm{ml}$ streptomycin, $100 \mathrm{U} / \mathrm{ml}$ penicillin and $0.29 \mathrm{mg} / \mathrm{ml}$ GlutaMAX; all from Invitrogen; Thermo Fisher Scientific, Inc.) containing different concentrations $(0-50 \mathrm{mg} / \mathrm{ml})$ of recombinant human BMP-2 (Medtronic, Dublin, Ireland). The cells were dyed with TRIzol reagent (Invitrogen; Thermo Fisher Scientific, Inc.) and then collected $72 \mathrm{~h}$ after BMP- 2 treatment. Total RNA was extracted using the TRIzol reagent, and the expression of Gremlin-1 mRNA was analyzed by reverse transcription-quantitative polymerase chain reaction (RT-qPCR). Gremlin-1 expression levels were initially standardized by $\beta$-actin, and then transformed to a ratio over the control $0 \mathrm{mg} / \mathrm{ml} \mathrm{BMP-2}$ treatment group. For the time-course study, human MSCs cultured in $35 \mathrm{~mm}$ tissue culture dishes were treated with or without $0.1 \mathrm{mg} / \mathrm{ml} \mathrm{BMP-2}$, and cell samples were collected at $12,24,48,72$ and $96 \mathrm{~h}$. Gremlin-1 expression levels were first standardized by $\beta$-actin, and then transformed to the ratio over the gremlin-1 expression levels at $0 \mathrm{~h}$.

$R T$-qPCR. Total RNA was quantified with a NanoDrop ND-1000 spectrophotometer (Thermo Fisher Scientific, Inc., Wilmington, DE, USA), and was reverse transcribed into cDNA with the iScript cDNA synthesis kit (Bio-Rad
Laboratories, Inc., Hercules, CA, USA). Reverse transcription was conducted in a $20 \mathrm{ml}$ reaction volume with the following protocol: $25^{\circ} \mathrm{C}$ for $5 \mathrm{~min}, 42^{\circ} \mathrm{C}$ for $30 \mathrm{~min}$ and $85^{\circ} \mathrm{C}$ for $5 \mathrm{~min}$. qPCR was conducted in quadruplicate using the iQ5 system (Bio-Rad Laboratories, Inc.). The reaction mixture had a volume of $25 \mathrm{ml}$ and contained $10 \mathrm{ng}$ cDNA, 1X iQ SYBR Green supermix (Bio-Rad Laboratories, Inc.) and $200 \mathrm{nM}$ of each primer.

The primer sequences used in this study were as follows: $\beta$-actin, forward 5'-CATGTACGTTGCTATCCAGGC-3' and reverse 5'-CTCCTTAATGTCACGCACGAT-3'; Gremlin-1, forward 5'-CGGAGCGCAAATACCTGAAG-3' and reverse 5'-GGTTGATGATGGTGCGACTGT-3'; ALP, forward 5'-GTGAACCGCAACTGGTACTC-3' and reverse 5'-GAG CTGCGTAGCGATGTCC-3'; OC, forward 5'-GAAGCCCAG CGGTGCA-3' and reverse, 5'-CACTACCTCGCTGCCCTC C-3'; IBSP, forward 5'-CACTGGAGCCAATGCAGAAGA-3' and reverse 5'-TGGTGGGGTTGTAGGTTCAAA-3'; OPN, forward 5'-CTCCATTGACTCGAACGACTC-3' and reverse 5'-CAGGTCTGCGAAACTTCTTAGAT-3'; MSX2, forward 5'-ATGGCTTCTCCGTCCAAAGG-3' and reverse 5'-CGG CTTCTTGTCGGACATGA-3'; RUNX2, forward 5'-TGGTTA CTGTCATGGCGGGTA-3' and reverse 5'-TCTCAGATCGTT GAACCTTGCTA-3'. qPCR was conducted with the following protocols: 1 cycle of $95^{\circ} \mathrm{C}$ for $3 \mathrm{~min}$, followed by 45 cycles of $95^{\circ} \mathrm{C}$ for $10 \mathrm{sec}, 58^{\circ} \mathrm{C}$ for $20 \mathrm{sec}$, and $72^{\circ} \mathrm{C}$ for $10 \mathrm{sec}$. All amplifications were normalized by $\beta$-actin. Data were analyzed using the comparison $\mathrm{Cq}\left(2^{-\Delta \Delta \mathrm{Cq}}\right)$ method (27) and expressed as fold change compared to respective control (28).

Assessment of gremlin-1 small interfering RNA (siRNA) efficacy. Four synthetic siRNAs (Qiagen, Inc., Valencia, CA, USA), which were designed to target multiple regions of human gremlin-1 mRNA (gene accession number: NM_001191322.1), were obtained and their sequence information is listed in Table I. Human MSCs were transfected with control siRNA and gremlin-1 siRNAs by Lipofectamine 2000 (Thermo Fisher Scientific, Inc.) under the optimized condition of transfection studied previously to validate the knockdown efficacy of the gremlin-1 siRNAs (28). In total, 6 groups were designed in triplicate: i) Non-transfected MSCs (NT control group); ii) MSCs transfected with control siRNA (control siRNA group); iii) MSCs transfected with gremlin-1 siRNA1 (siRNA1 group); iv) MSCs transfected with gremlin-1 siRNA2 (siRNA2 group); v) MSCs transfected with gremlin-1 siRNA3 (siRNA3 group); and vi) MSCs transfected with gremlin-1 siRNA4 (siRNA4 group). Following transfection for $24 \mathrm{~h}, \mathrm{MSC}$ were transferred to the basal medium containing $0.1 \mathrm{mg} / \mathrm{ml}$ BMP-2. Total RNA was extracted $72 \mathrm{~h}$ following treatment with BMP-2 using TRIzol reagent according to the manufacturer's protocols and gremlin-1 mRNA expression was assessed by RT-qPCR. Gremlin-1 protein expression levels in the culture supernatant were calculated using a commercial enzyme linked immunosorbent assay kit for human gremlin-1 (EK1292; Wuhan Uscn Business Co., Ltd., Wuhan, Hubei, China) $72 \mathrm{~h}$ after treatment with BMP-2. The most effective gremlin-1 siRNA to inhibit gremlin-1 mRNA and protein expression levels was selected for the following studies. To examine the duration of gremlin-1 inhibition resulting from a single transfection of gremlin-1 siRNA in the presence of 
BMP-2, a time-course study in which gremlin-1 expression was analyzed following siRNA transfection was performed. Initially, samples were collected to measure the baseline of gremlin-1 expression (day 0) prior to transfection. For the transfection, three groups were constructed in triplicate: One group without transfection (NT control); one group transfected with control siRNA (control siRNA); and one group transfected with the most effective gremlin-1 siRNA. The medium was replaced after a $24 \mathrm{~h}$ period of transfection with basal medium containing $0.1 \mathrm{mg} / \mathrm{ml} \mathrm{BMP}-2$, and this medium was replaced twice a week. Cell samples were collected on days 1, 3, 7, 10 and 14. Total RNA was extracted using TRIzol reagent according to manufacturer's protocols at the indicated time points to perform RT-qPCR, in order to assess the mRNA expression levels of gremlin-1 at these time points.

Water soluble tetrazolium salt-8 (WST-8) assay. Human MSCs were inoculated in 48-well plates in quadruplicate with a density of $5.5 \times 10^{3}$ cells $/ \mathrm{cm}^{2}$. Following culture for $24 \mathrm{~h}$, human MSCs in $300 \mathrm{ml}$ medium were transfected with control siRNA or the most effective gremlin-1 siRNA. The medium was altered $24 \mathrm{~h}$ later with basal medium containing $0.1 \mathrm{mg} / \mathrm{ml}$ BMP-2. The metabolism of human MSCs was estimated on days 1, 3 and 7. The WST-8 (2-(2-methoxy-4-nitrophenyl)-3(4-nitrophenyl)-5-(2,4-disulfophenyl)-2H-tetrazolium, monosodium salt; Cedarlane Laboratories Ltd., Burlington, Canada) reagent is designed to estimate the activity of mitochondrial dehydrogenase in human MSCs. WST- 8 is decreased by mitochondrial dehydrogenases in viable cells to produce a soluble yellow formazan, which is directly proportional to the reduced activity of the cells, and thus is used as a measurement of total cellular metabolic activity. WST-8 solution $(30 \mu \mathrm{l})$ was added to $300 \mu \mathrm{l}$ medium containing human MSCs $\left(1.0 \times 10^{4}\right)$, and incubated for a further $3 \mathrm{~h}$. Following this, the absorbance was measured at $450 \mathrm{~nm}$ with an absorbance microplate reader (BioTek Instruments, Inc., Winooski, VT, USA) and the reference wavelength was $650 \mathrm{~nm}$. The ratios between the data of individual patients and the non-transfection group were calculated and then the data of all five patients were combined.

DNA content analysis. The human MSCs were inoculated in 96-well plates in quadruplicate at a density of $3 \times 10^{3}$ cells $/ \mathrm{cm}^{2}$. Following culture for $24 \mathrm{~h}$, human MSCs were transfected with control siRNA or the most effective gremlin-1 siRNA. The medium was replaced with basal medium with $0.1 \mathrm{mg} / \mathrm{ml}$ BMP-2 24 h later. Cells were dyed using a CyQUANT Cell Proliferation Assay kit (Invitrogen; Thermo Fisher Scientific, Inc.) and samples were gathered on days 0,3 and 6. The DNA content was measured with an emission wavelength of $530 \mathrm{~nm}$ and an excitation wavelength of $450 \mathrm{~nm}$. The ratios between the data of individual patients and the non-transfection group were first calculated and then data of all 5 patients were combined.

Induction of osteogenic differentiation of human MSCs. To estimate the influence of gremlin-1 inhibition on osteogenic differentiation in human MSCs, gremlin-1 expression was knocked down in human MSCs, and human MSCs were induced to perform osteogenic differentiation in osteogenic medium [DMEM with $10 \% \mathrm{FBS}, 100 \mathrm{mg} / \mathrm{ml}$ streptomycin, $100 \mathrm{U} / \mathrm{ml}$ penicillin, $0.29 \mathrm{mg} / \mathrm{ml} \mathrm{L-glutamine} \mathrm{(all} \mathrm{from}$
Invitrogen; Thermo Fisher Scientific, Inc.), $5 \mathrm{mM}$ b-glycerolphosphate, $10 \mathrm{nM}$ dexamethasone, and $50 \mathrm{mg} / 1$ ascorbic acid-2-phosphate (all from Sigma-Aldrich, Merck KGaA)] which contained $0.1 \mathrm{mg} / \mathrm{ml}$ BMP-2 in 12-well plates. Three study groups were constructed: One group with no transfection (NT control); one group transfected with control siRNA (control siRNA); and one group transfected with the most effective gremlin-1 siRNA. All three groups were treated with osteogenic medium with $0.1 \mathrm{mg} / \mathrm{ml}$ BMP-2 $24 \mathrm{~h}$ post transfection. siRNA transfection was repeated every 7 days, as advised by the manufacturer (Qiagen, Inc.) to cause prolonged silencing of gremlin-1. The following experiments were conducted to assess osteogenic differentiation

RT-qPCR analysis of osteoblastic genes. On day 14 of osteogenic induction, cell samples were gathered, total RNA was extracted using TRIzol reagent and reverse transcription was conducted as described above. Expression levels of osteoblastic genes including alkaline phosphatase (ALP), msh homeobox 2 (MSX2), integrin-binding sialoprotein (IBSP), osteocalcin (OC), runt related transcription factor 2 (RUNX2) and osteopontin (OPN) were detected by RT-qPCR. Human $\beta$-actin, used as the endogenous reference gene, standardized the osteoblastic gene expression. Following standardization, the ratio between the data of individual patients and the non-transfection group were first calculated and then data of all 5 patients were combined. Gremlin-1 expression levels were also measured to confirm the inhibition of gremlin-1 on day 14 .

ALP staining. On day 14 of osteogenic induction, ALP staining was conducted using a Fast Naphthol phosphate kit (Sigma-Aldrich; Merck KGaA). Cells were washed three times using PBS and were subsequently fixed for $30 \mathrm{sec}$ by citrate-acetone-formaldehyde fixative. Samples were then rinsed with deionized water in order to be stained in the dark for $15 \mathrm{~min}$, and counterstained with neutral red solution for 2 min (Fig. 5). Samples were then washed three times using tap water to remove the dissociative dye. The experiment was repeated three times independently.

ALP activity assay. On day 14 of osteogenic induction, the activity of ALP was quantitatively measured using a commercial phosphatase assay kit (BioAssay Systems, Hayward, CA, USA). Lysis buffer (containing $50 \mathrm{mM}$ Tris-HCl, $0.5 \%$ Triton and $5 \mathrm{mM} \mathrm{MgCl}_{2}$; Sigma-Aldrich; Merck KGaA) was used to lyse the cells in triplicate. The lysate was then moved to 96-well plates and incubated with ALP substrate at $37^{\circ} \mathrm{C}$ for $30 \mathrm{~min}$ in the dark. Stop buffer $(0.1 \mathrm{M} \mathrm{NaOH})$ was then added to halt the reaction. The p-nitrophenol product, which was generated by enzymatic hydrolysis of p-nitrophenylphosphate substrate was detected at $405 \mathrm{~nm}$ using an absorbance microplate reader (BioTek Instruments, Inc.). The protein concentration of samples was tested using a detergent compatible protein assay kit (Bio-Rad Laboratories, Inc.) and FBS (Bio-Rad Laboratories, Inc.), and a standard curve was created to transform data. Concentration of ALP was standardized by total protein amount. The ratio between the data of individual patients and the non-transfection group were first calculated and then the data of all 5 patients were combined. 
Table I. Sequences of the four synthetic siRNAs targeting gremlin-1 mRNA.

\begin{tabular}{|c|c|c|c|}
\hline siRNA no. & Target position & Target sequence $\left(5^{\prime}-3^{\prime}\right)$ & RNA oligo sequences ( $\left.5^{\prime}-3^{\prime}\right)$ \\
\hline siRNA1 & $477-499$ & TCGTTGCATATCCATCGATTTGG & $\begin{array}{l}\text { AAAUCGAUGGAUAUGCAACGA } \\
\text { GUUGCAUAUCCAUCGAUUUGG }\end{array}$ \\
\hline siRNA2 & $559-581$ & GACCTAAAACAACCAGATTCTTA & $\begin{array}{l}\text { AGAAUCUGGUUGUUUUAGGUC } \\
\text { CCUAAAACAACCAGAUUCUUA }\end{array}$ \\
\hline siRNA3 & $989-1011$ & TCCATCTCTTCTTAAGTTGATAG & $\begin{array}{l}\text { AUCAACUUAAGAAGAGAUGGA } \\
\text { CAUCUCUUCUUAAGUUGAUAG }\end{array}$ \\
\hline siRNA4 & $1218-1240$ & AAGCTTGAAAGGCCAATACCAGA & $\begin{array}{l}\text { UGGUAUUGGCCUUUCAAGCUU } \\
\text { GCUUGAAAGGCCAAUACCAGA }\end{array}$ \\
\hline
\end{tabular}

siRNA, small interfering RNA.

Alizarin Red staining. On day 28 of osteogenic induction, cells and the extracellular matrix from all groups were fixed in $75 \%$ ethanol at $48^{\circ} \mathrm{C}$ for $1 \mathrm{~h}$, washed with distilled water, and stained with Alizarin Red S solution (Sigma-Aldrich; Merck KGaA) until an orange-red color appeared (Fig. 6). Samples were then rinsed three times with deionized water, and washed once with PBS.

Calcium assay. On day 28 of osteogenic induction, $600 \mathrm{ml}$ $0.5 \mathrm{~N}$ hydrochloric acid solution was added to each well (12-well plates) to demineralize the cells and the extracellular matrix secreted by the cells, which were then incubated at $48^{\circ} \mathrm{C}$ overnight. Following centrifugation of the samples at $10,000 \times \mathrm{g}$ for $10 \mathrm{~min}$, the supernatant containing calcium extracts was gathered. The calcium concentration was examined using the QuantiChrom Calcium Assay kit (BioAssay Systems). The ratio between the data of individual patients and the non-transfection group were first calculated and then the data of all 5 patients were combined.

Statistical analysis. Data are presented as the mean \pm standard error and were analyzed by Student's $t$-test or one-way analysis of variance followed by the Bonferroni post hoc test, as appropriate. All tests were two-tailed, with $\mathrm{P}<0.05$ considered to indicate a statistically significant difference. All statistical analyses were conducted with PASW Statistics version 18.0 (SPSS Inc., Chicago, IL, USA).

\section{Results}

BMP-2 induces gremlin-1 mRNA expression. Gremlin-1 mRNA expression was increased by BMP-2, and the peak expression appeared when induced with $1 \mathrm{mg} / \mathrm{ml}$ BMP-2 (Fig. 1A). Increasing concentrations of BMP-2 resulted in increasing gremlin-1 mRNA expression levels within the range from $0.01-1 \mathrm{mg} / \mathrm{ml}$ BMP-2, whereas the induction of gremlin-1 expression was reduced as the concentration of BMP-2 rose from 1 to $50 \mathrm{mg} / \mathrm{ml}$ (Fig. 1A). There was no significant difference in the level of gremlin-1 expression between the group without BMP-2 treatment and the group treated with $50 \mathrm{mg} / \mathrm{ml}$ BMP-2 (P>0.05; Fig. 1A).

Gremlin-1 mRNA expression was also induced by $0.1 \mathrm{mg} / \mathrm{ml}$ BMP-2 in a time-dependent manner. Gremlin-1
mRNA expression levels increased with time and at 24, 48, 72 and $96 \mathrm{~h}$ following BMP-2 treatment the increase was significantly higher than the group without BMP-2 treatment $(\mathrm{P}<0.05, \mathrm{P}<0.01, \mathrm{P}<0.01$ and $\mathrm{P}<0.01$, respectively; Fig. $1 \mathrm{~B})$.

Suppression of BMP-2-induced gremlin-1 expression by gremlin-1 siRNA transfection. Following the examination of all transfection conditions, the optimal condition was $20 \mathrm{nM}$ (final concentration) of siRNA duplex with $3 \mu$ l Lipofectamine RNAiMAX in $1 \mathrm{ml}$ medium for each well of the 12-well tissue culture plates, which produced the highest mean fluorescence intensity and the highest percentage of siRNA-positive cells in the transfected cells. The following siRNA transfection studies were performed under this optimal condition. Compared with the non-transfected group, gremlin-1 mRNA (Fig. 2A) and protein (Fig. 2B) levels were not changed by transfection with control siRNA. By contrast, gremlin-1 mRNA expression levels in human MSCs and gremlin-1 protein levels in the culture supernatant were significantly reduced by the transfection of gremlin-1 siRNA1 $(\mathrm{P}<0.01$ and $\mathrm{P}<0.01$, respectively; Fig. 2A and B, respectively) and siRNA2 $(\mathrm{P}<0.01$ and $\mathrm{P}<0.01$, respectively; Fig. $2 \mathrm{~A}$ and $\mathrm{B}$, respectively). Gremlin-1 mRNA and protein expression were not altered by transfection of gremlin-1 siRNA3 and siRNA4 (Fig. 2A and B). As gremlin-1 mRNA and protein expression levels were significantly more inhibited with gremlin-1 siRNA1 compared with gremlin-1 siRNA1 $(\mathrm{P}<0.05$ and $\mathrm{P}<0.05$, respectively; Fig. $2 \mathrm{~A}$ and $\mathrm{B}$, respectively), further experiments were conducted with gremlin-1 siRNA2 only. A single gremlin-1 siRNA2 transfection significantly inhibited BMP-2 induced gremlin-1 expression on days 3, 7 and 10 compared with the untransfected group and the control siRNA group, but the inhibitive effect was not evident on day 14 (Fig. 2C).

Increased viability of human MSCs by gremlin-1 suppression. Following siRNA transfection, the WST-8 assay indicated that metabolism of MSCs was significantly increased by transfection with gremlin-1 siRNA2 on days 3 and 7 compared with the untransfected group and the control siRNA group $(\mathrm{P}<0.01$; Fig. 3A). The total protein content in the group transfected with gremlin-1 siRNA2-was also significantly higher compared with the untransfected group and the control siRNA group on day 14 ( $\mathrm{P}<0.05$; Fig. 3B). In addition, following siRNA transfection, 
A

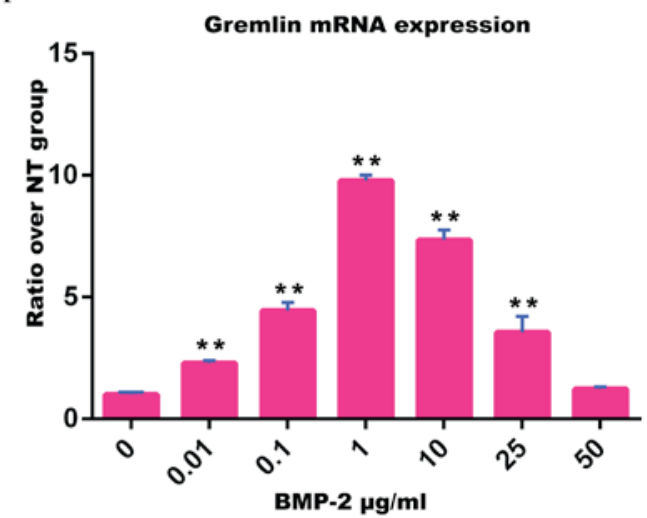

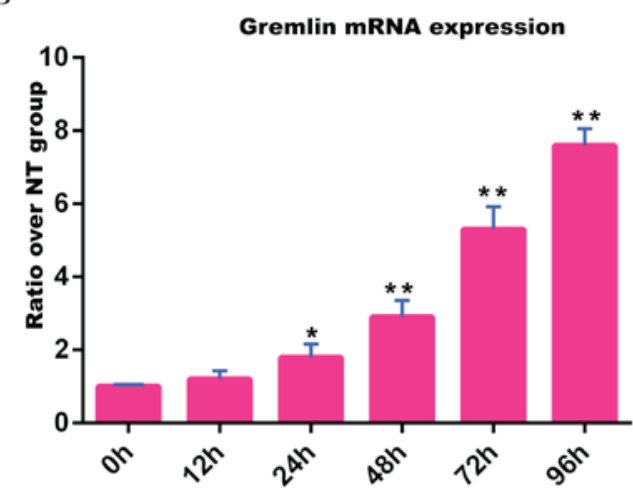

Figure 1. Gremlin-1 mRNA expression is induced by BMP-2 in human mesenchymal stem cell cultures. (A) The variable effect of BMP-2 dose. ** P $<0.01$ vs. the untreated control. (B) The time-dependent effect of $0.1 \mathrm{mg} / \mathrm{ml} \mathrm{BMP}-2$ treatment. ${ }^{*} \mathrm{P}<0.05$ and ${ }^{* * *} \mathrm{P}<0.01 \mathrm{vs} .0 \mathrm{~h}$. BMP, bone morphogenetic protein.

A

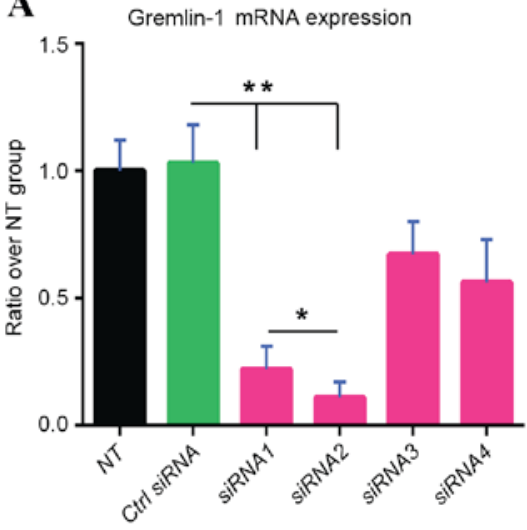

B

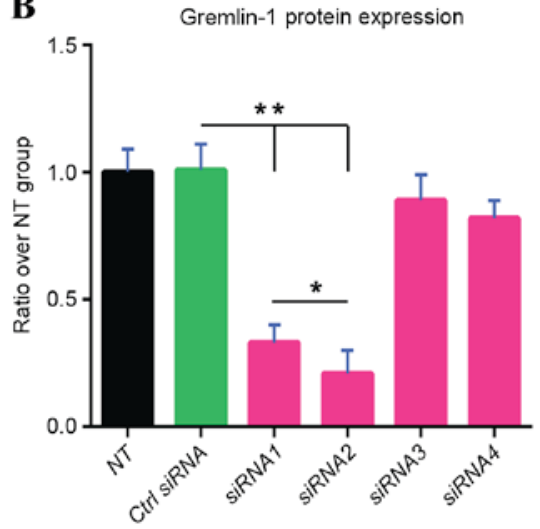

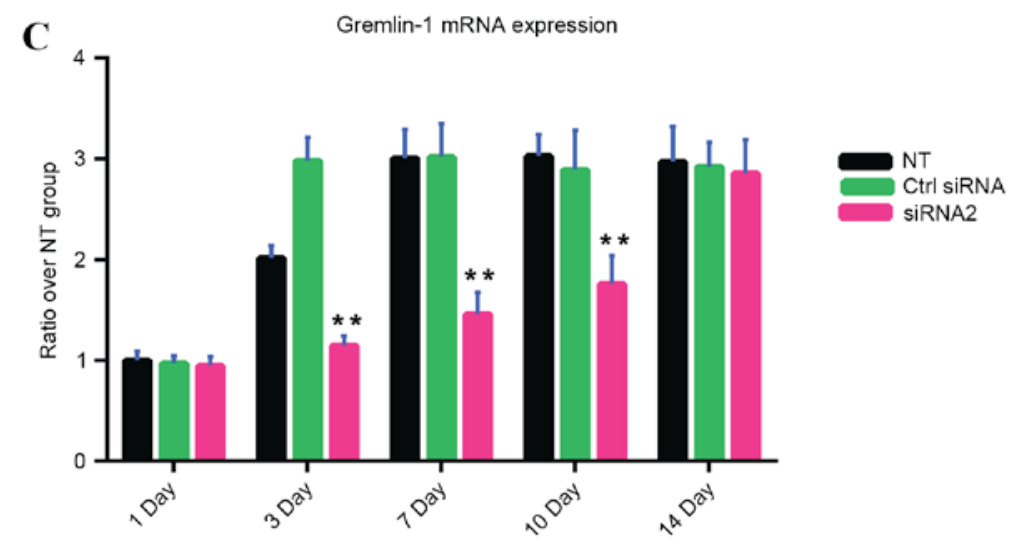

Figure 2. Efficacy of Gremlin-1 siRNAs in suppressing gremlin-1 expression. (A) Gremlin-1 mRNA and (B) protein expression $72 \mathrm{~h}$ after transfection with different siRNAs. ${ }^{*} \mathrm{P}<0.05$ and ${ }^{* *} \mathrm{P}<0.01$, with comparisons indicated by brackets. (C) Gremlin-1 mRNA expression levels in human mesenchymal stem cells treated with siRNA2 over time. ${ }^{*} \mathrm{P}<0.05$ and ${ }^{* *} \mathrm{P}<0.01$ vs. NT and Ctrl siRNA groups at the same time point. siRNA, small interfering RNA; NT, not transfected; Ctrl, control.

the total DNA content was significantly increased by gremlin-1 siRNA 2 on days 3 and 7 compared with the untransfected group and the control siRNA group (day $3, \mathrm{P}<0.01$ and $\mathrm{P}<0.01$, respectively; day $7, \mathrm{P}<0.05$ and $\mathrm{P}<0.05$, respectively; Fig. $3 \mathrm{C}$ ).

Increased osteogenic differentiation of human MSCs by gremlin-1 suppression. siRNA transfection was performed every 7 days during the osteogenic differentiation of human MSCs, as the inhibitive effect of a single gremlin-1 siRNA2 transfection lasted for a maximum of 7 days (Fig. 2C). Gremlin-1 siRNA2 significantly increased the mRNA expression levels of all osteoplastic genes examined, including ALP ( $\mathrm{P}<0.01$; Fig. 4A), IBSP $(\mathrm{P}<0.01$; Fig. 4B), MSX2 ( $<<0.01$; Fig. 4C), OC ( $\mathrm{P}<0.05$; Fig. 4D), OPN ( $\mathrm{P}<0.01$; Fig. 4E) and 

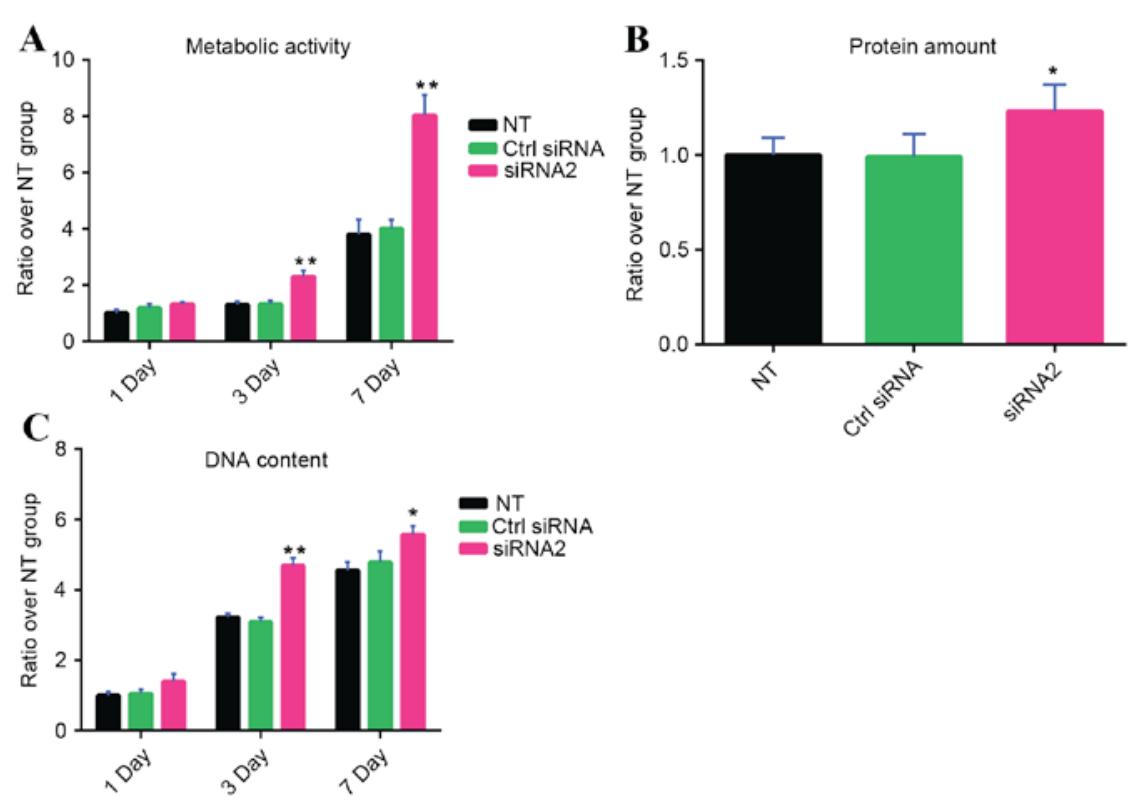

Figure 3. Increased viability of human mesenchymal stem cells following gremlin-1 suppression. (A) Metabolism, (B) protein content and (C) DNA content of human MSCs following gremlin-1 interference. ${ }^{*} \mathrm{P}<0.05$ and ${ }^{* *} \mathrm{P}<0.01$ vs. NT and Ctrl siRNA groups. siRNA, small interfering RNA; NT, not transfected; Ctrl, control.
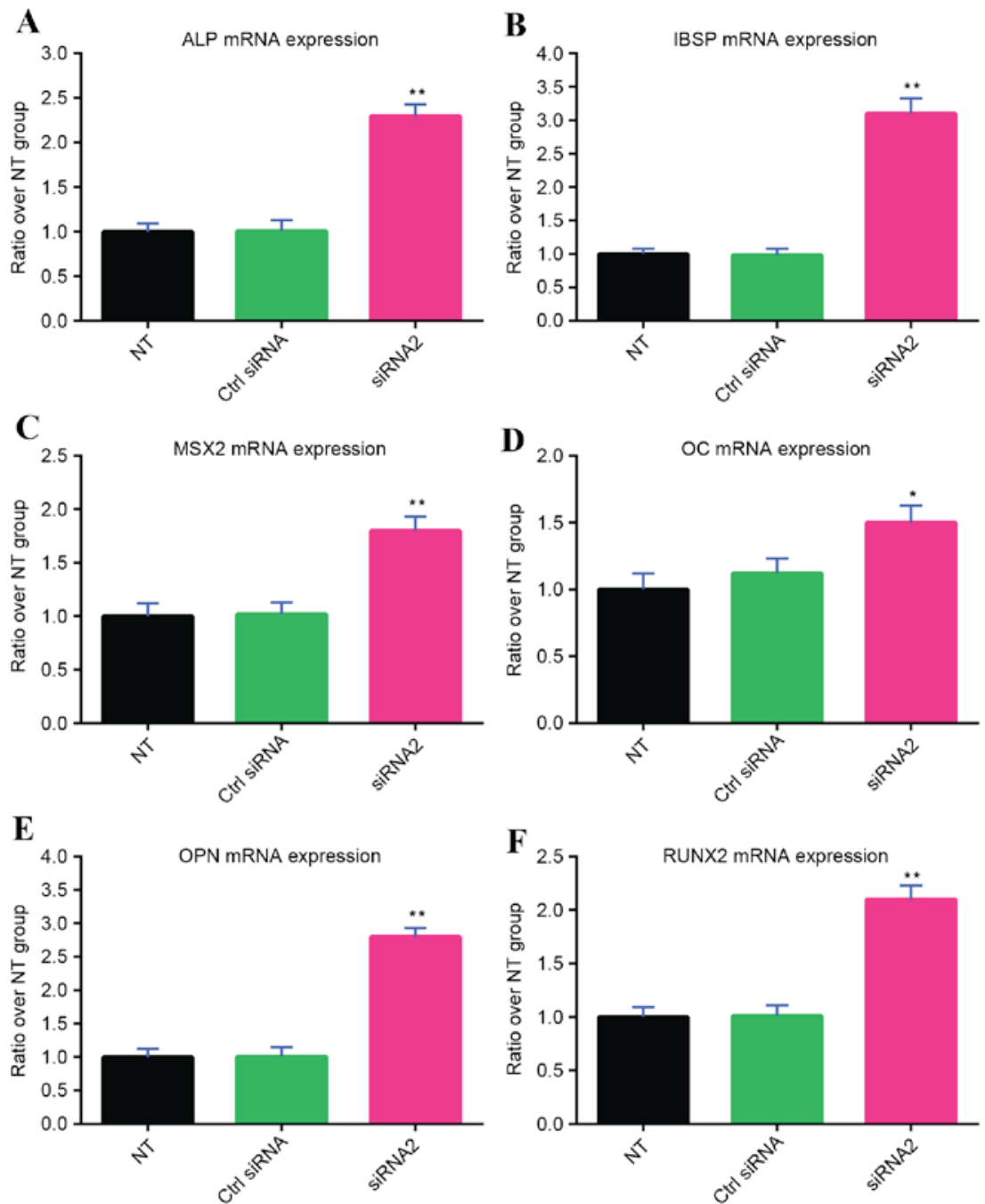

Figure 4. Expression levels of the osteoblastic genes (A) ALP, (B) IBSP, (C) MSX2, (D) OC, (E) OPN and (F) RUNX2 on day 14 of osteogenic induction by osteogenic medium containing $0.1 \mathrm{mg} / \mathrm{ml}$ BMP-2. ${ }^{*} \mathrm{P}<0.05$ and ${ }^{* *} \mathrm{P}<0.01$ vs. NT and Ctrl siRNA groups. ALP, alkaline phosphatase; IBSP, integrin-binding sialoprotein; MSX2, msh homeobox 2; OC, osteocalcin; OPN, osteopontin; RUNX2, runt related transcription factor 2; siRNA, small interfering RNA; NT, not transfected; Ctrl, control. 
A
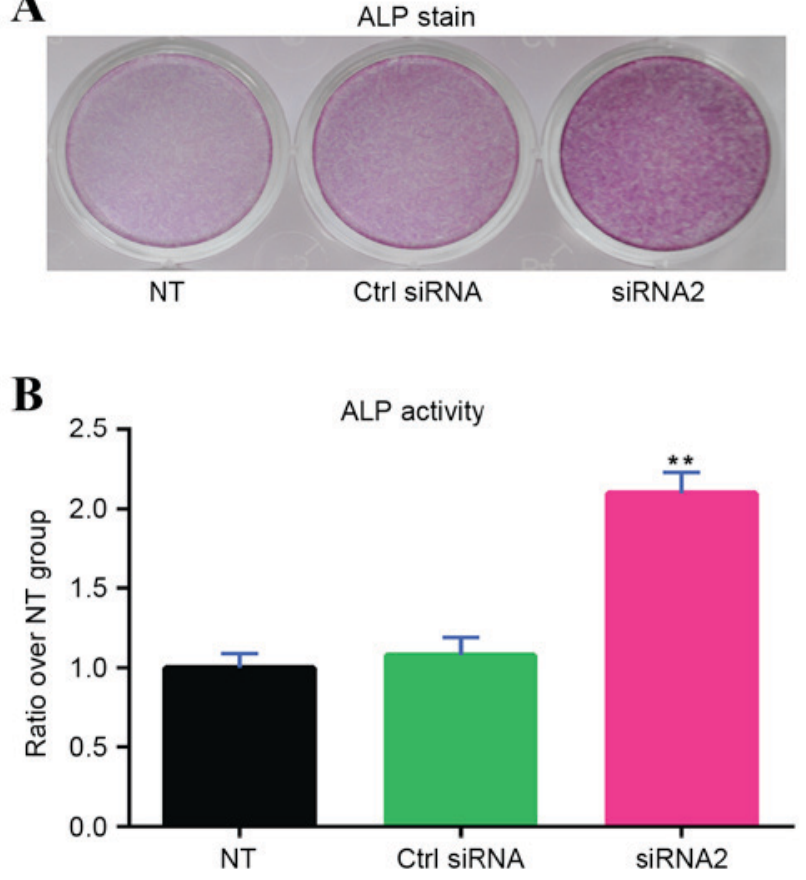

Figure 5. ALP activity was increased by gremlin-1 suppression, as determined by (A) fast Naphthol phosphate staining and (B) enzymatic ALP assay on day 14 of osteogenic induction by osteogenic medium containing $0.1 \mathrm{mg} / \mathrm{ml}$ BMP-2. ${ }^{* *} \mathrm{P}<0.01$ vs. NT and Ctrl siRNA groups. ALP, alkaline phosphatase; siRNA, small interfering RNA; NT, not transfected; Ctrl, control.

\section{$\mathbf{A}$}
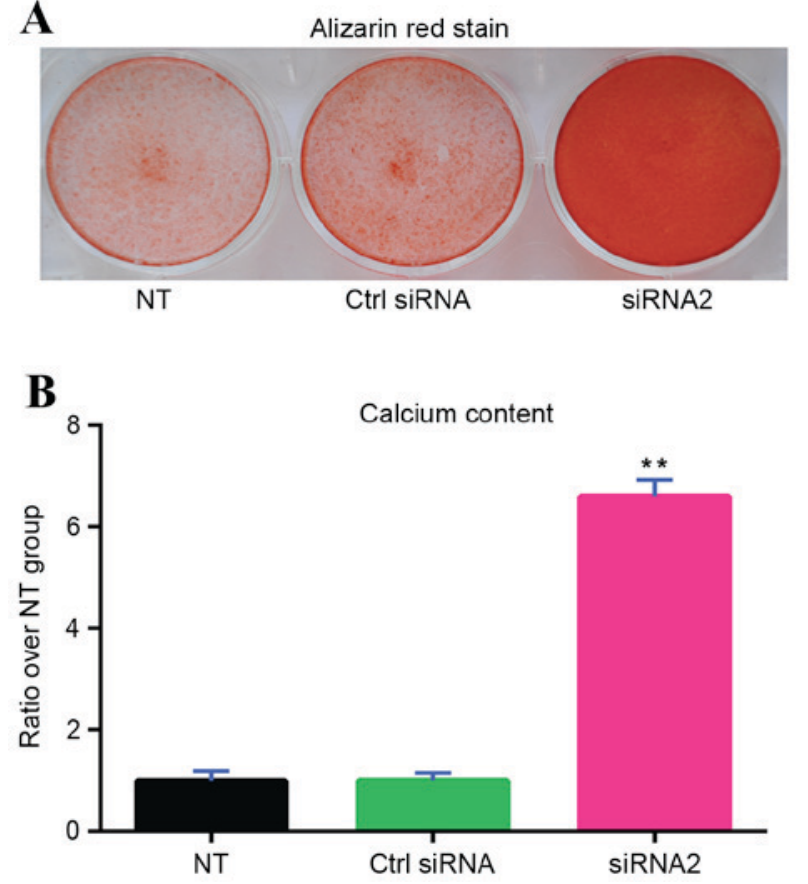

Figure 6. Calcium deposits were increased by gremlin-1 suppression, as determined by (A) Alizarin Red staining and (B) calcium assay on day 28 of osteogenic induction by osteogenic medium containing $0.1 \mathrm{mg} / \mathrm{ml}$ BMP-2. ${ }^{* *} \mathrm{P}<0.01$ vs. NT and Ctrl siRNA groups. siRNA, small interfering RNA; NT, not transfected; Ctrl, control.

RUNX2 ( $\mathrm{P}<0.01$; Fig. 4F) compared with the untransfected group and the control siRNA group. The qualitative naphthol phosphate staining test indicated that following 14 days of osteogenic induction, transfection of gremlin-1 siRNA2 increased the ALP activity of the cells (Fig. 5A). Similar results were obtained in the quantitative ALP assay, which revealed that transfection of gremlin-1 siRNA2 significantly increased ALP activity of the MSCs compared with the untransfected group and the control siRNA group ( $\mathrm{P}<0.01$; Fig. $5 \mathrm{~B})$.

Qualitative Alizarin Red staining indicated that compared with the untransfected group and the control siRNA group, more calcium deposits were observed in the group transfected with gremlin-1 siRNA2 (Fig. 6A). The results of the quantitative calcium assay demonstrated that gremlin-1 siRNA2 transfection significantly increased calcium deposits in MSCs compared with the untransfected group and the control siRNA group $(\mathrm{P}<0.01$; Fig. $6 \mathrm{~B})$.

\section{Discussion}

The results of the present study suggested that gremlin-1 inhibition improved viability and osteogenic differentiation of human MSCs by BMP-2-inducement. These results add to the understanding of the involvement of gremlin-1 in human MSC osteogenesis, and imply that gremlin-1 may inhibit bone formation in humans. In human MSC cultures, gremlin-1 expression was induced by BMP-2 in experiments of dose- and time-dependence. To the best of our knowledge, the present study is the first to observe the biophysical nature of time-dependent gremlin-1 induction by BMP-2, and the variable effect depending on the dose. The induction of gremlin-1 was enhanced by BMP-2 within the concentrations from $0.01-1 \mathrm{mg} / \mathrm{ml}$, although the induction was decreased at concentrations of BMP-2 from 1-50 mg/ml (Fig. 1). However, the reason why BMP-2 concentrations $>1 \mathrm{mg} / \mathrm{ml}$ reduces gremlin-1 induction remains to be elucidated. DNA content, cellular metabolism and protein content of human MSCs were increased by gremlin-1 inhibition, and this influence was unlikely to result from the transfection system as the transfection of control siRNA did not change these features of human MSCs in culture (Fig. 3A-C). These data suggest that gremlin-1 is involved in proliferation of stem cells. Although the data indicated that gremlin-1 inhibition increased the viability of human MSCs, the expression levels of osteoblastic genes and the increased ALP induction on day 14 may have been the secondary effect of gremlin-1 inhibition on cell viability, as expression levels of osteoblastic genes in the present study were standardized by $\beta$-actin from the matching group, and the concentration of ALP was standardized by the total protein amount. An increase in osteogenic activity was still apparent following such standardization, so the differences cannot be explained by stagnated cell growth alone. The expression levels of osteoblastic genes and increased ALP production by gremlin-1 inhibition indicated increased osteogenic differentiation on a per cell basis. Nevertheless, the number of calcium deposits was not standardized, and therefore the enhanced calcium deposits caused by gremlin-1 inhibition may be partly due to increased cell growth. The inhibition of gremlin-1 expression by a single transfection of gremlin-1 siRNA2 in the present study was maintained for a maximum of 7 days (Fig. 2C). Based on this observation, gremlin-1 siRNA2 was used to transfect cells every 7 days during the period of osteogenic induction, so as to knock 
down the expression of gremlin-1 constantly. Repeated transfections of gremlin-1 siRNA2 continuously inhibited gremlin-1 expression.

Instead of studying the influence of exogenous gremlin-1 addition, the present study illustrated the influence of endogenous gremlin-1 on BMP-2-induced osteogenesis in human MSCs via the knockdown of endogenous gremlin-1 expression. The different observations between studies using human MSCs and rodent cells or animal models indicate that a species-specific difference in BMP-2-induced osteogenesis-associated functions of gremlin-1 in MSCs may exist. Dexamethasone and BMP-2 resulted in different osteoinductive effects on MSCs of humans, rats, and mice (29). Rat MSCs expressed mRNA for activin receptor-like kinase-6, a type 1 receptor for BMPs, but MSCs from humans did not express this particular receptor (30). In human MSCs, MSX2 was upregulated up to 10-fold by BMP-2, but in rat MSCs expression of MSX2 was not altered by BMP-2 (30). These observations and the data from the present study suggest that a species-specific difference may exist when gremlin-1 functions in BMP-2-induced osteogenesis of MSCs.

The present study indicated that in the presence of BMP-2, gremlin-1 is harmful to osteogenic differentiation in human MSCs. One of the possible mechanisms may be that BMP-2 is not inactivated by gremlin-1 that is already bound to BMP-2 in human MSC cultures. Gremlin-1 is known to bind to proteoglycans on the surface of cell. In addition, regarding the possible intracellular signaling, based on the following observations gremlin-1 may partly regulate osteogenic differentiation by modulating the intracellular canonical BMP/Smad signaling, then MSX2, RUNX2, or other unidentified transcription factors, followed by the subsequent gene expression of $\mathrm{OC}$, IBSP, OPN and ALP. A previous report (31) has demonstrated that gremlin-1 actively inhibits BMP-2 and the production of its type 2 receptor. The data from the present study suggested that gremlin-1 inhibition increased the expression of MSX2 and RUNX2, which are important transcription factors in the process of osteogenic differentiation. In addition, the expression levels of the osteoblastic markers IBSP, OC, ALP and OPN were all increased by gremlin-1 inhibition (Fig. 4). Further studies are required regarding the intracellular signaling pathways through which gremlin-1 regulates osteogenic differentiation in human MSCs.

In conclusion, expression of gremlin-1 in human MSCs was upregulated by BMP-2 in a time-dependent manner, with the effect of dose being variable. Gremlin-1 inhibition increased BMP-2-induced osteogenic differentiation and viability in human MSC culture, which indicates that gremlin-1 has an inhibitory effect on human MSC osteogenesis. The underlying mechanisms that resulted in these observations remain unknown, and further studies are needed to clarify the intracellular signaling pathways through which gremlin-1 regulates osteogenic differentiation in human MSCs. In addition, similar studies with a larger sample size on human MSCs are important to discover whether there is any significant difference between different sexes in response to gremlin-1 inhibition.

\section{Acknowledgements}

The present study was supported by a grant from the Anhui Natural Science Foundation (grant no. 1408085MH182).

\section{References}

1. Granero-Moltó F, Weis JA, Miga MI, Landis B, Myers TJ, O'Rear L, Longobardi L, Jansen ED, Mortlock DP and Spagnoli A: Regenerative effects of transplanted mesenchymal stem cells in fracture healing. Stem Cells 27: 1887-1898, 2009.

2. Marie PJ and Fromigué O: Osteogenic differentiation of human marrow-derived mesenchymal stem cells. Regen Med 1: 539-548, 2006.

3. Koehler KC, Alge DL, Anseth KS and Bowman CN: A Diels-Alder modulated approach to control and sustain the release of dexamethasone and induce osteogenic differentiation of human mesenchymal stem cells. Biomaterials 34: 4150-4158, 2013.

4. Yokota J, Chosa N, Sawada S, Okubo N, Takahashi N, Hasegawa T, Kondo $\mathrm{H}$ and Ishisaki A: PDGF-induced PI3K-mediated signaling enhances the TGF- $\beta$-induced osteogenic differentiation of human mesenchymal stem cells in a TGF- $\beta$-activated MEK-dependent manner. Int J Mol Med 33: 534-542, 2014.

5. Tsuji K, Bandyopadhyay A, Harfe BD, Cox K, Kakar S, Gerstenfeld L, Einhorn T, Tabin CJ and Rosen V: BMP2 activity, although dispensable for bone formation, is required for the initiation of fracture healing. Nat Genet 38: 1424-1429, 2006.

6. Lin L, Fu X, Zhang X, Chen LX, Zhang JY, Yu CL, Ma KT and Zhou CY: Rat adipose-derived stromal cells expressing BMP4 induce ectopic bone formation in vitro and in vivo. Acta Pharmacol Sin 27: 1608-1615, 2006.

7. Kotajima S, Kishimoto KN, Watanuki M, Hatori M and Kokubun S: Gene expression analysis of ectopic bone formation induced by electroporatic gene transfer of BMP4. Ups J Med Sci 111: 231-241, 2006.

8. Kugimiya F, Kawaguchi H, Kamekura S, Chikuda H, Ohba S, Yano F, Ogata N, Katagiri T, Harada Y, Azuma Y, et al: Involvement of endogenous bone morphogenetic protein (BMP) 2 and BMP6 in bone formation. J Biol Chem 280: 35704-35712, 2005.

9. Tsuji K, Cox K, Gamer L, Graf D, Economides A and Rosen V: Conditional deletion of BMP7 from the limb skeleton does not affect bone formation or fracture repair. J Orthop Res 28: 384-389, 2010.

10. Levet S, Ciais D, Merdzhanova G, Mallet C, Zimmers TA, Lee SJ, Navarro FP, Texier I, Feige JJ, Bailly S and Vittet D: Bone morphogenetic protein 9 (BMP9) controls lymphatic vessel maturation and valve formation. Blood 122: 598-607, 2013.

11. Kang Q, Song WX, Luo Q, Tang N, Luo J, Luo X, Chen J, Bi Y, He BC, Park JK, et al: A comprehensive analysis of the dual roles of BMPs in regulating adipogenic and osteogenic differentiation of mesenchymal progenitor cells. Stem Cells Dev 18: 545-559, 2009.

12. Shi Y and Massagué J: Mechanisms of TGF-beta signaling from cell membrane to the nucleus. Cell 113: 685-700, 2003.

13. Foletta VC, Lim MA, Soosairajah J, Kelly AP, Stanley EG, Shannon M, He W, Das S, Massague J and Bernard O: Direct signaling by the BMP type II receptor via the cytoskeletal regulator LIMK1. J Cell Biol 162: 1089-1098, 2003.

14. Lavery K, Swain P, Falb D and Alaoui-Ismaili MH: BMP-2/4 and BMP-6/7 differentially utilize cell surface receptors to induce osteoblastic differentiation of human bone marrow-derived mesenchymal stem cells. J Biol Chem 283: 20948-20958, 2008.

15. Ali IH and Brazil DP: Bone morphogenetic proteins and their antagonists: Current and emerging clinical uses. Br J Pharmacol 171: 3620-3632, 2014.

16. Passa O, Tsalavos S, Belyaev NN, Petryk A, Potocnik AJ and Graf D: Compartmentalization of bone morphogenetic proteins and their antagonists in lymphoid progenitors and supporting microenvironments and functional implications. Immunology 134: 349-359, 2011.

17. Yanagita M: Antagonists of bone morphogenetic proteins in kidney disease. Curr Opin Investig Drugs 11: 315-322, 2010.

18. Dean DB, Watson JT, Moed BR and Zhang Z: Role of bone morphogenetic proteins and their antagonists in healing of bone fracture. Front Biosci (Landmark Ed) 14: 2878-2888, 2009.

19. Hsu DR, Economides AN, Wang X, Eimon PM and Harland RM: The Xenopus dorsalizing factor Gremlin identifies a novel family of secreted proteins that antagonize BMP activities. Mol Cell 1: 673-683, 1998.

20. Topol LZ, Bardot B, Zhang Q, Resau J, Huillard E, Marx M, Calothy G and Blair DG: Biosynthesis, post-translation modification, and functional characterization of Drm/Gremlin. J Biol Chem 275: 8785-8793, 2000. 
21. Karagiannis GS, Musrap N, Saraon P, Treacy A, Schaeffer DF, Kirsch R, Riddell RH and Diamandis EP: Bone morphogenetic protein antagonist gremlin-1 regulates colon cancer progression. Biol Chem 396: 163-183, 2015.

22. Khokha MK, Hsu D, Brunet LJ, Dionne MS and Harland RM: Gremlin is the BMP antagonist required for maintenance of Shh and Fgf signals during limb patterning. Nat Genet 34: 303-307, 2003.

23. Lappin DW, McMahon R, Murphy M and Brady HR: Gremlin: An example of the re-emergence of developmental programmes in diabetic nephropathy. Nephrol Dial Transplant 17 (Suppl 9): S65-S67, 2002.

24. Bardot B, Lecoin L, Fliniaux I, Huillard E, Marx M and Viallet JP: Drm/Gremlin, a BMP antagonist, defines the interbud region during feather development. Int J Dev Biol 48: 149-156, 2004.

25. Michos O, Panman L, Vintersten K, Beier K, Zeller R and Zuniga A: Gremlin-mediated BMP antagonism induces the epithelial-mesenchymal feedback signaling controlling metanephric kidney and limb organogenesis. Development 131: 3401-3410, 2004.
26. Gazzerro E, Smerdel-Ramoya A, Zanotti S, Stadmeyer L, Durant D, Economides AN and Canalis E: Conditional deletion of gremlin causes a transient increase in bone formation and bone mass. J Biol Chem 282: 31549-31557, 2007.

27. Livak KJ and Schmittgen TD: Analysis of relative gene expression data using real-time quantitative PCR and the 2(-Delta Delta C(T)) Method. Methods 25: 402-408, 2001.

28. Zuo B, Zhu J, Li J, Wang C, Zhao X, Cai G, Li Z, Peng J, Wang P, Shen C, et al: microRNA-103a functions as a mechanosensitive microRNA to inhibit bone formation through targeting Runx2. J Bone Miner Res 30: 330-345, 2015.

29. Diefenderfer DL, Osyczka AM, Reilly GC and Leboy PS: BMP responsiveness in human mesenchymal stem cells. Connect Tissue Res 44 (Suppl 1): S305-S311, 2003.

30. Osyczka AM, Diefenderfer DL, Bhargave G and Leboy PS: Different effects of BMP-2 on marrow stromal cells from human and rat bone. Cells Tissues Organs 176: 109-119, 2004.

31. Wellbrock J, Harbaum L, Stamm H, Hennigs JK, Schulz B, Klose H, Bokemeyer C, Fiedler W and Lüneburg N: Intrinsic BMP antagonist gremlin-1 as a novel circulating marker in pulmonary arterial hypertension. Lung 193: 567-570, 2015. 